\title{
Theory of Resonances in Molecular Systems
}

\author{
R. Lefebvre, Orsay \\ (Laboratoire de Photophysique Moléculaire du CNRS)
}

\begin{abstract}
Resonances are met in many aspects of molecular dynamics such as photo- and predissociation of molecules and complexes, unimolecular decay of polyatomic species and reactive scattering. Recent advances in the theory of resonances has led to a better understanding of the nature of resonant states in terms of associating to them quantum numbers reflecting the appropriate (sometimes unobvious) degrees of freedom.
\end{abstract}

The resonance concept is present in all fields of physics. An unstable particle is a manifestation of the resonance phenomenon. To take another example in a completely different area, a surface plasmon in a solid can be described as a damped solution of Maxwell's equations and the word resonance is again appropriate. An intense activity has been recently devoted to this concept in the field of molecular physics. This is due primarily to the use of experimental devices (essentially atomic or molecular beams and lasers) which allow for a considerable improvement in the quality of the information obtained from a study of collisions or half-collisions involving atoms and molecules. The measured energy, time and angular distributions require often the consideration of the resonances which mediate the processes. There has been simultaneously considerable progress toward a rigorous theory of resonance states which are described by solutions of the wave equation under a special type of boundary conditions. Molecular physics with its large variety of interacting entities and forms of potential gives many opportunities of applying these new tools. A favourable circumstance in this undertaking is the existence in quantum molecular physics and quantum chemistry of a tradition of high numerical accuracy in the manipulation of large basis sets and the integration of coupled differential equations. This review is not centred on the experimental manifestations of the resonance phenomenon in molecular

physics but deals with recent advances in the theory which were initiated by consideration of some molecular problems. In this varied and fast developing subject some selection has to be made which is naturally guided by personal interests. Thus no mention will be made of recent important advances which involve electron motion (such as electronmolecule scattering or photoionization). We shall concentrate on molecular (or heavy particle) motion in a few atom system.

\section{When are we finding resonance?}

After centre of mass separation, the wave equation of an atomic or molecular system is said to possess solutions corresponding to either bound states or scattering states. In a bound state the particles (electrons and nuclei) are constrained to remain in a finite region of configuration space. The scattering states allow for the description of collision processes. The situation is in fact not as clear-cut as this. All excited bound states are subject to radiative decay. It may also happen that the bound character is the result of an approximation and the real system is able to dissociate by the loss of electrons (ionization) or of atomic or molecular subunits (dissociation). Even the ground state is not stable since collision with a photon may lead to dissociation. Thus bound states are just a zero ${ }^{\text {th }}$ order view of decaying states, that is resonances. On the other hand, collision experiments lead to the determination of energy or

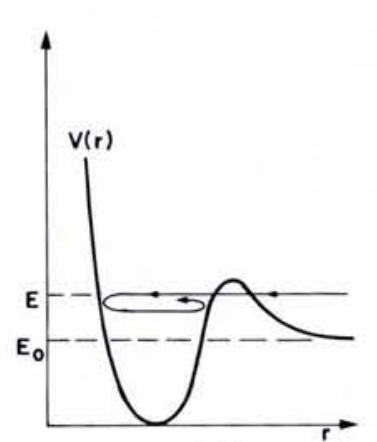

(a)

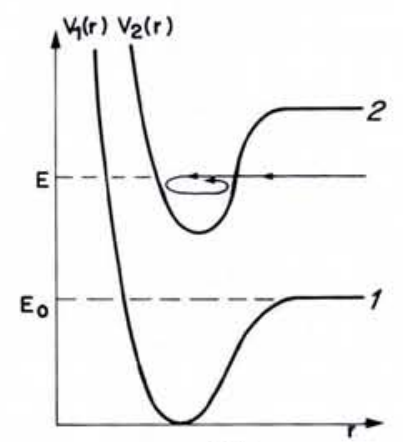

(b)
Fig. 1 - One and twochannel description of resonances. In both cases an incident wave associated with an open channel can reach the well, either by tunnelling through the barrier, or because of channel interaction which splits the wave into wavelets. The corresponding resonances are either called shape (case (a)) or Feshbach (case (b)). angle-dependent cross-sections which are more or less structured. Sharp features in a cross-section are usually associated with the production of long-lived intermediates. The characterization of these unstable species also leads to the consideration of resonances. Thus we may say that, whether obvious or unobvious, resonance identification is of concern in most atomic or molecular studies.

\section{Which Definition for a Resonance?}

Whatever definition is adopted for a resonance there is always the idea that a complex energy is involved, which has the form $E=E,-i \Gamma_{t} / 2$. Since the frequency factor of a wave function is $\exp (-(\mathrm{i} / \hbar) E t)$ such an energy produces in the density (squared modulus of the wave function) a factor $\exp (-\Gamma, t / \hbar)$. We can therefore associate with a resonance a lifetime $\tau_{r}=\hbar / \Gamma_{r}$ and a decay rate $K_{r}=\Gamma_{r} / \hbar$. However the preparation of a resonant state and the observation of an exponential time dependence of the decay requires generally this resonance to be well isolated from others. Two resonances are said to be isolated (or non-overlapping) when the difference in the real parts of their complex energies largely exceeds their respective imaginary parts (to be called in short their widths).

Most of the traditional approaches to the resonance problem consist in solving the wave equation for real energies, this being supplemented by some procedure to determine the width. One merit of recent developments is the possibility that is given of reaching directly their complex eigen-energies, even in conditions where other approaches are bound to fail. We will start by recalling some of the real energy procedures.

There is a formal tool which is almost universally present in problems of molecular dynamics: the coupled channel approach. The degrees of freedom of the system are separated into two types: the coordinate (a distance) which describes the relative motion of two entities and all other coordinates. The relative motion is usually the difficult part of the problem. For instance in a collision between an atom and a diatomic molecule, we can assume we know how to describe vibration of the diatom and the rotations (either of the diatom or of the system as a whole). This information is used to write the complete wave function in the form

$$
\psi(\mathbf{q}, r)=\sum_{n} \varphi_{n}(\mathbf{q}, r) U_{n}(r)
$$

where $r$ is the inter-fragment coordinate (from atom to centre of mass of the diatom in this example), $\mathbf{q}$ denotes col- 
lectively all remaining coordinates and $n$ is a label referring to the known information. The dependence of the $\varphi_{n}{ }^{\prime} s$ on $r$ allows for the possibility of having the internal motion depending on the relative position (adiabaticity). One term in the series may sometimes provide already a reasonable description of the dynamics. The equation for the relevant function, say $U(r)$, is found to have the form of the radial Schrödinger equation. Retention of two terms leads to two coupled differential equations. One may in this way build one-, two-, ... n-channel descriptions of the dynamics.

Examples of potentials which may correspond to one or two channels are shown in Fig. 1. Although a solution of the wave equation exists for an arbitrary energy above threshold energy $E_{0}$, at some particular energies the back and forth reflection of an incident wave reaching the well may lead to constructive interference. The large amplitude which results can be made the basis for a method for determining resonance energies (still real at this stage of the work). From one resonance to the next above in energy, one additional node will be present in the wave function in the region of the well. In the two cases depicted in Fig. 1, the wave function $U(r)$ behaves for large $r$ as $C(k) \sin [k r+\delta(E)]$ with $k^{2} \sim E$. A change by $\pi$ of the quantity $\delta(E)$ (the phase shift) indicates a change in nodal structure. Still another definition is based upon looking at the maxima in the time delay given by $\tau_{\mathrm{d}}=$ $2 \hbar d \delta / d E$. This is the additional time it takes for a wave packet to move across the interaction region as compared to free motion. All these definitions are easy to apply for isolated resonances and in practice they give practically identical results. Each has its own recipe for calculating the width.

Turning now to the methods which lead directly to the complex energies, we start from the observation that in the asymptotic region, $U(r)$ is proportional to a combination of two exponentials (describing the ingoing and outgoing waves):

$U(r) \sim[-\exp (-i k r)+S(k) \exp (i k r)]$ The scattering amplitude $S(k)=\exp$ $(2 \mathrm{i} \delta)$ is a number of modulus unity for a real energy since $\delta$ is real. However we can try to look for values of $k$ (which have to be complex) such that the outgoing term dominates the ingoing term. An extreme situation is a value $k_{r}$ of $k$ such that $S\left(k_{r}\right)$ is infinite $\left(k_{r}\right.$ is said to be a pole of $S(k)$, that is $S(k)$ has a factor $\left.\left(k-k_{r}\right)^{-1}\right)$. A class of these poles is found to be of the form $k_{r}=k_{0}-i k_{1}$ with $k_{0}$ and $k_{1}$ positive. We have there-
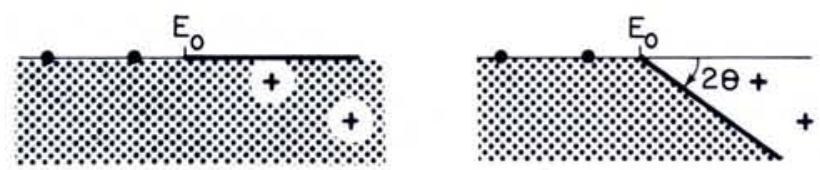

Fig. 2 - Left: schematic spectrum of a one-channel Hamiltonian. $E_{0}$ is the threshold energy and the thick segment symbolizes the continuum of scattering states. The resonance energies marked by crosses are "hidden". They cannot be calculated just by letting E be of the form $E_{,}-i \Gamma_{r} / 2$.

Right: spectrum of the rotated Hamiltonian. The continuum is rotated clockwise and the resonance energies are now visible. Two bound state energies marked by dots are unaffected by the operation.

fore the possibility of defining a wave function which has a purely outgoing character, with an eigen-energy which is a complex number. This is the definition of resonant states given by Siegert, which is of course not limited to our simple situation with only one open channel (that is with only one channel with a threshold below collision energy). The wave function for large $r$ behaves as $\exp [i k r]=\exp \left[i k_{0} r\right] \exp \left[k_{1} r\right]$.

The divergence at infinity is the behaviour expected for a decaying state: observation of the density (or current) at a distance $r$ corresponds to breaking events which occurred in the past at the origin, when there was more 'activity' in the source.

In some rare cases, $S(k)$ is known analytically so that the resonance energies are immediately available, but the more common situation is to define approximations yielding analytical forms for $S(k)$ (many of the semi-classical treatments are of this type). Finally there is the task of determining as accurately as possible the resonance energies once a form for the wave function has been chosen. The complex rotation method described in the next section provides a very efficient and by now very popular method for doing this.

\section{Complex Rotation and Complex Eigen- values}

Briefly speaking, the complex rotation technique amounts to looking at the consequences of substituting $\rho \exp (i \theta)$ (with $\rho$ real) instead of $r$ into the wave equation. The effect of rotation can be appreciated by starting from the form of the wave function in the asymptotic region when the coordinate is real. The function with a wave number of the form $k_{0}-i k_{1}$ can also be written as $U(r) \sim A(k) \exp \left[-i k_{0} r\right] \exp \left[-k_{1} r\right]$ $+B(k)\left[\exp i k_{0} r\right] \exp \left[k_{1} r\right]$.

Resonance states correspond to choosing $k$ in such a way that only the outgoing term is left. This means trying to detect the vanishing of the ingoing component, which is normally dominated (because of the factor $\exp \left[-k_{1} r\right]$ ) by the diverging outgoing component. Let us write $k$ in the form $K \exp (-i \beta)$ with $\tan \beta$ $=k_{1} / k_{0}$. After complex rotation, the wave function becomes

$$
\begin{aligned}
U \rho \exp (\mathrm{i} \theta) \sim & A(k) \exp [-i K \rho \cos (\theta-\beta)] \\
& \exp [K \rho \sin (\theta-\beta)] \\
+ & B(k) \exp [-\mathrm{i} K \rho \cos (\theta-\beta)] \\
& \exp [-K \rho \sin (\theta-\beta)]
\end{aligned}
$$

If $\sin (\theta-\beta)>0$ the unwanted (ingoing) component is now increasing so that its detection is made easier. The outgoing component on the other hand is decreasing as $\rho \rightarrow+\infty$. This situation is the same as that met for a bound state. Beyond the relevant turning point, the bound state wave function for an arbitrary energy has both growing and decaying components. The quantized energies are those energies which lead to disappearance of the growing term.

This effect of using a complex variable to solve second order linear differential equations with outgoing boundary conditions was already exploited by Hartree during World War II in a study of radio wave propagation. The method was rediscovered years later in quantum mechanics, first applied in a heuristic way and then put on a firm mathematical basis for certain classes of potentials. The theory examines the spectral properties of the transformed Hamiltonian. Its spectrum is compared to that of the untransformed Hamiltonian in Fig. 2 in the single channel case. The important point is that the continuum (the energies of the scattering states) which starts at the threshold energy $E_{0}$ is rotated clockwise by angle $2 \theta$, so that the resonant energies which are below the real axis are now discrete eigenenergies of the new Hamiltonian. The consequence is that their eigenfunctions are localized. This localization opens the route to two alternatives. The first procedure consists of developing the resonant wave function on a basis of localized functions (harmonic oscillator functions, gaussians, etc.). The solution of the wave equation is then replaced by the diagonalization of the Hamiltonian matrix. Because of truncation effects with a finite matrix the eigenvalues are found all to depend on $\theta$. However it is observed that some of them pause at some particular values of $\theta$ corresponding to the 


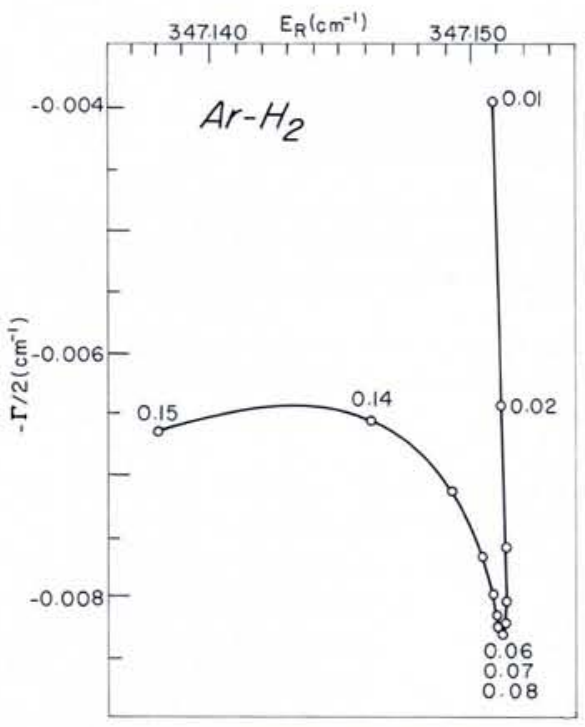

Fig. 3 - A $\theta$-trajectory calculated for the $\mathrm{Ar}-\mathrm{H}_{2}$ van der Waals complex. Two potentials disposed as those of Fig. 1(b) are involved, with the diatom either in its lower or third rotational state. The energy is stabilized for $\theta$ close to $0.07 \mathrm{rad}$. The width read on the imaginary axis can be directly correlated with the spectral broadening mechanism called rotational predissociation.

fulfilment of the condition $\partial E / \partial \theta=0$. It is reasonable to identify these energies as being those of resonant states. Fig. 3 gives an example of such a dependence of the energy upon the rotation angle (a so-called $\theta$-trajectory) for a van der Waals complex (see J. Reuss and S. Stolte, EN, January (1985) p. 9).

A different procedure consists in a step by step numerical integration of the radial Schrödinger equation (or of the coupled channel equations) with introduction of the appropriate boundary conditions. In a radial problem these conditions are vanishing at the origin and an asymptotic behaviour which should be derived from the Siegert boun-

Fig. 4 - The string of resonances associated with the potential $10 r^{2} \exp (-0.5 r)$. The vertical dashed line shows the position of the top of the barrier. Integer quantum numbers can be associated rigorously with these resonances.

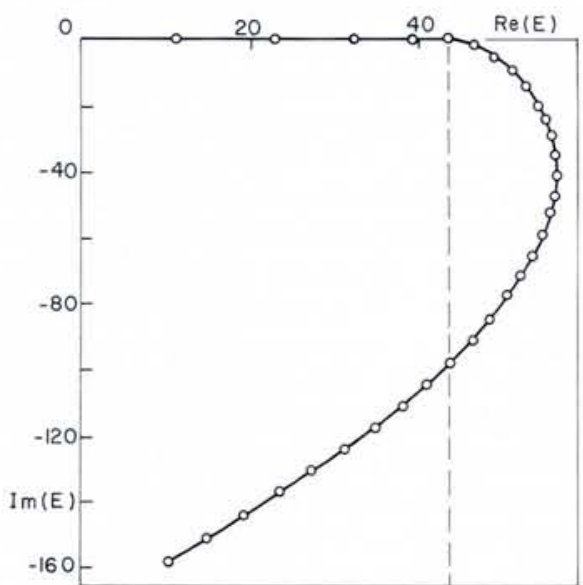

dary condition. The energy is to be adjusted until there is continuity of the wave function and of its derivative. This technique can be shown to be very powerful and accurate. One may, for example, investigate the resonances of a repulsive exponential potential $A$ $\exp (-\alpha r)$ with $A, \alpha>0$, a case hard to approach in terms of constructive interference effects. One may prove also that to a given potential there corresponds generally a rich spectrum of resonant states. As an example, Fig. 4 gives a so-called 'string of resonances'. The resonance energies may have imaginary parts which exceed considerably their real parts. Here again the strict application of a boundary condition produces results which go far beyond a simple intuitive picture. Such resonance states are however not as useful are those of the more common type, although they should be used in reconstructing the scattering amplitude from its poles.

\section{Quantum Labelling the Resonant States}

An interesting by-product of complex rotation is the possibility of associating quantum numbers in a rigorous way with the resonances of the one channel problem. For bound states it is possible to introduce an auxiliary function $W(r)$ obeying a nonlinear differential equation (Milne's equation) in such a way that for $h \rightarrow 0$, one has the correspondence $W^{-2}(r) \rightarrow k(r)$. The quantity $W^{-2}(r)$ has sometimes been called quantum momentum. A rigorous quantization condition for motion on the $r$ axis from $-\infty$ to $+\infty$ is

$$
\int_{-\infty}^{+\infty} W^{-2}(r) d r=(n+1) \pi, n=0,1, \ldots .
$$

The analogy with the (approximate) Bohr-Sommerfeld rule is striking; $n+1$ instead of $n+1 / 2$ accounts for motion beyond the classical turning points. For resonant states the theory can be similarly developed after the wave equation has been written in terms of the variable $\rho \exp (i \theta)$. A function $\widetilde{W}[\rho \exp (i \theta)]$ is to be calculated first and a rigorous quantization condition of the same form with $\widetilde{W}$ in place of $W$ is obtained which associates successive integers to the resonant states of a given potential (for instance to the resonances belonging to the string displayed in Fig. 4).

No rigorous method exists for labelling the resonances when several degrees of freedom are involved. A simple case is that of moderately broadened Feshbach resonances where it is natural to label the resonances with the quantum numbers of the bound states. Weakly bound van der Waals complexes provide a good example. For the less obvious cases semi-classical procedures have been developed which extend to the resonances an argument used for bound states. This consists in determining approximate constants of the motion which allow for the application of quantization rules similar to the Bohr-Sommerfeld one. Finally advantage can be taken of the fact that there is an inherent flexibility in the coupled channel approach. One may look for a system of coordinates which would decouple as much as possible the channels so as to identify shape or Feshbach resonances. This idea has been successfully applied to the analysis of resonances detected in reactive scattering calculations (not to speak of experiments where the subject is still in its infancy). The characterization of resonances in this case amounts to a study of the transition state of the theoretical chemists and it has even been suggested that this may lead some day to a 'spectroscopy of the transition state'.

\section{Stabilization Graphs and their Analyti- cal Continuation}

We end this review, by pointing out that the link between real and complex energy approaches can be made in a very suggestive way through the socalled stabilization graphs. We have seen that at a (real) resonance energy the wave function has a large amplitude in the interior region. The matrix of the Hamiltonian can be diagonalized in a basis of integrable functions. If the basis functions are made to depend on a scaling factor (such as the parameter $\eta$ of an exponential function $\exp (-\eta r))$ variation of this parameter is somewhat equivalent to changing the size of a box which encloses the system. The function approximating the resonance state is much less sensitive to this change than are the functions approximating the scattering states. Fig. 5 gives a typical diagram of energies versus the scaling parameter. The resonant state shows up as the stable root (hence the expression stabilization graph), which however is subjected to the non crossing rule. The avoided crossings indicate proximity of a complex value of $\eta$ for which $\partial E / \partial \eta=0$, with $E$ complex. Since a complex scaling factor $\eta=\eta_{0} \exp [i \theta]$ is equivalent to the use of the variable $\eta_{0} r$ $\exp [i \theta]$, we are back to the complex rotation method (or at least a variant of it). This procedure has been successfully applied to the determination of resonance energies (and therefore of decay rates) in systems of coupled oscillators which may simulate the behaviour of 
small polyatomic molecules with a large excess of vibrational energy. An interesting output of such calculations was the demonstration that even at these high energies there may exist resonant states with widely different life-times. This is important for the theory of unimolecular decay since this shows that we may sometimes expect so-called mode-specificity in internal vibrational redistribution of energy.

\section{Suggested Reading}

Resonances in Electron-Molecule Scattering, van der Waal's Complexes and Reactive Chemical Dynamics, ed. D.G. Truhlar, ACS Symposium Series, 263 (1984).
Fig. 5-A stabiliza- E tion graph for the potential $7.5 r^{2} \exp (-r)$. The eigenvalues of a matrix are plotted as a function of a (real) scaling parameter. The avoided crossings can be exploited to determine the resonance energy.

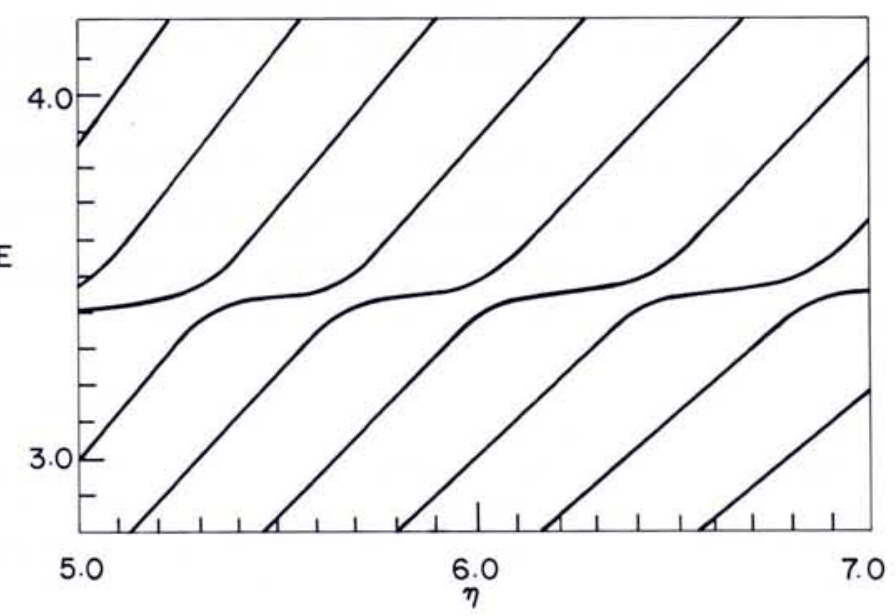

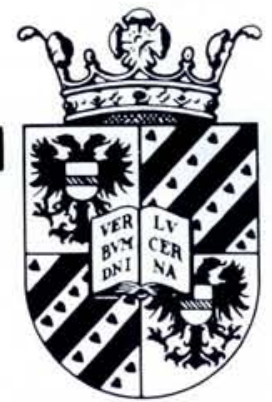

\section{university of groningen the netherlands asks:}

\section{professor/senior-scientist $\mathrm{m} / \mathrm{f}$ in atomic physics}

(vac.nr. 850101/2493)

at the Department of Physics.

The appointed candidate will be associated with the Kernfysisch Versneller Instituut (K.V.I.) in Groningen.

The K.V.I. in Groningen is a national center for Nuclear Physics research that is jointly sponsored by the University of Groningen and the Foundation for Fundamental Research on Matter, F.O.M. The regular staff of the K.V.I. consists of 75 persons, of which 35 are scientists.

In addition to the research in nuclear physics with the light and heavy ion beams of the $\mathrm{K}=160 \mathrm{MeV}$ cyclotron of the K.V.I., there is an active program in atomic physics and related subjects.

This research is based mainly on the use of beams of highly ionised atoms which are directly obtained from an ECR ion source. It is performed in close collaboration with groups from outside the K.V.I. At this moment there are two experimental set-ups available for studying electron capture processes in ion-atom collisions, and one for studying the interaction of slow, highly ionised atoms with surfaces. In addition there is at the K.V.I. research on stepped surfaces by scattering of low-energy ions (LEIS).
The successful applicant is supposed to take on the daily responsibility for existing research in atomic physics and to initiate new programs in atomic physics or related fields using the research facilities of the K.V.I. He will participate in the teaching activities of the Department of Physics.

The appointment will carry a maximum salary of $f$ 7.996,- per month (Dutch Civil Servants Code).

Applications, including a curriculum vitae, a list of publications and the names of three referees, should be addressed to the Director of Personnel, Rijksuniversiteit Groningen, P.O. Box 72, $9700 \mathrm{AB}$ Groningen (The Netherlands), within a period of 4 weeks after date of publication, quoting reference number 850101 .

Further information on this position can be obtained from the chairman of the search committee: prof.dr. A. van der Woude, K.V.I., telephone number The Netherlands, 50-115736.

Those wishing to draw attention to potential candidates are welcome to contact the committee chairman. 